\title{
Energy enhancement and spectrum narrowing in terahertz electron sources due to negative mass instability
}

\author{
Yu. Lurie, ${ }^{1, *}$ V. L. Bratman, ${ }^{1,2}$ and A. V. Savilov ${ }^{2}$ \\ ${ }^{1}$ Ariel University, Ariel \\ ${ }^{2}$ Institute of Applied Physics, Russian Academy of Sciences, 603950 Nizhny Novgorod, Russia
}

(Received 10 March 2016; published 26 May 2016)

\begin{abstract}
Simulations of coherent spontaneous undulator radiation in a waveguide demonstrate that the use of negative mass instability (NMI) for retaining longitudinal sizes of dense electron bunches, which are formed in laser-driven photoinjectors, allows one to increase power capabilities of a terahertz radiation source by many times. The NMI is realized in an undulator with combined helical and over-resonance uniform longitudinal magnetic fields due to nonisochronous longitudinal oscillations of electrons, whose frequencies increase/decrease with increasing/decreasing particle energy. In such conditions, an effective longitudinal size of the bunches can be preserved at long distance even at an extremely high electron density. Correspondingly, an energy extraction efficiency of more than $20 \%$ is revealed at a narrow frequency radiation spectrum, suggesting realization of a compact and powerful $\mathrm{THz}$ source.
\end{abstract}

DOI: 10.1103/PhysRevAccelBeams.19.050704

\section{INTRODUCTION}

Terahertz electromagnetic radiation is currently of increasing interest for basic science and many promising applications. Various ways for generation in the $\mathrm{THz}$ frequency range have been already developed. Among others, a powerful coherent Doppler-frequency-up-shifted radiation from preformed short ultrarelativistic electron bunches in undulators, which was proposed many years ago by Ginzburg [1] and Motz [2], is still fairly attractive. This method was very popular in the 1950s and later, however, its frequency possibilities were essentially limited because of the problems with formation of dense electron bunches, whose effective longitudinal sizes should be smaller than the radiation wavelength. These problems are overcome in free-electron lasers (FELs), where bunches are self-consistently formed, like in other electron devices. However, a possibility to realize the original ideas [1,2] arose also $[3,4]$ after development of modern laser-driven photoinjector linear accelerators that allowed preforming of dense picosecond and subpicosecond bunches.

A powerful source of coherent spontaneous $\mathrm{THz}$ radiation seems to be especially attractive when electron bunches are accelerated only in a photoinjector (without additional accelerating sections) up to a moderate energy being of the order of magnitude of 3-6 MeV. The final performing of the bunch can then (or simultaneously) be done in a short dispersive section. This method meets,

\footnotetext{
”ylurie@ariel.ac.il
}

Published by the American Physical Society under the terms of the Creative Commons Attribution 3.0 License. Further distribution of this work must maintain attribution to the author $(s)$ and the published article's title, journal citation, and DOI. however, strict limitations because of very strong Coulomb repulsion and fast longitudinal spreading of dense bunches consisting of "too light" electrons. These limitations can partially be mitigated by means of using preliminarily energy-chirped $[5,6]$ or spatially modulated $[7,8]$ bunches. However, significantly higher energy enhancement and spectrum narrowing can be expected [9] if a helical undulator with a very strong (over-resonance) guiding field is used and, thus, the conditions for nonisochronous longitudinal electron oscillations [10-17] are fulfilled. In this case, development of the negative mass instability can lead to formation of a long-living electron "core" with a sufficiently small longitudinal size. The effect has been already proved theoretically for the simplest onedimensional model of the electron bunch moving in a transversely uniform undulator field and radiating in free space [9]. In the present work, a more realistic threedimensional geometry for electron bunches, the undulator field and the operating $\mathrm{THz}$ waveguide has been considered. The simulations are carried out for the bunch parameters which are close to the expected parameters of the Israeli $\mathrm{THz}$ radiation source being constructed at Ariel University [18,19]. A laser-driven photocathode rf gun with the electron energy of up to $6 \mathrm{MeV}$ is supposed to be used as an electron source in this project. The 3-D spacefrequency approach [20] is used for numerical analysis of the radiation emission and bunch dynamics. The method is based on an expansion of the rf electromagnetic field in terms of eigenmodes of the medium in which the field is excited and propagates. The interaction between the field and the particles is described by a set of coupled equations for wave excitation and particle dynamics in the selfconsistent field, expressing the evolution of mode amplitudes along the interaction region. The method was realized 
in the numerical code WB3D and has been successfully applied to the analysis of various effects in FELs (see [6] and references therein).

The negative mass instability in undulators $[10,13-15]$ is similar to the classical negative mass instability (NMI) first discovered in cyclic accelerators [21,22] and then studied also for cyclotron resonance masers $[23,24]$. Some peculiarities of these instabilities are discussed in Sec. II of the present paper. Section III contains a brief description of the method used for WB3D simulations and the results of the simulations. In Sec. IV we discuss the capabilities of an advanced THz source, which could be realized on the basis of a laser-driven photocathode gun in Ariel University.

\section{NONISOCHRONOUS ELECTRON OSCILLATIONS AND NEGATIVE MASS INSTABILITY}

The classical version of the NMI discovered in cyclic accelerators [21,22] causes an effective phase (azimuthal) attraction of electrons rotating along Larmor circles in the uniform magnetic field $\mathbf{B}_{0}$ and mutually repulsing due to their quasistatic Coulomb interaction. This effect occurs due to the so-called nonisochronity of electron rotation caused by a specific relativistic dependence, $\frac{\partial \Omega_{c}}{\partial \gamma}<0$, of the electron cyclotron frequency $\Omega_{c}=e B_{0} / m c \gamma$ on particle energy $E=m c^{2} \gamma$. The corresponding decrease or increase in the cyclotron frequency with an increase or decrease in the electron energy caused by additional particle acceleration in the Coulomb field of an electron density fluctuation, can lead to attraction of the electron to the fluctuation and growth of the latter. A very similar mechanism (but with replacement of the Coulomb accelerating field by a synchronously rotating component of the rf field of an electrodynamic system) ensures the main cyclotron resonance maser (CRM) instability, azimuthal particle bunching on helical trajectories and predominance of stimulated radiation over absorption almost in all varieties of cyclotron resonance masers including their simplest and most effective version in the $\mathrm{THz}$ frequency range-gyrotron; the "conventional" NMI also develops in these devices along with the CRM instability, and ensures, in particular, an additional gain in gyroamplifiers [23,24].

The NMI in undulators can be realized in a combined undulator and uniform longitudinal magnetic field [9,10,13-15]. It is well known that electrons can move in such a field along the helical stationary trajectories with the normalized transverse velocity,

$$
\beta_{\perp}=\frac{K}{\gamma|\Delta|},
$$

where $K=e B_{u} \lambda_{u} / 2 \pi m c^{2}$ is the undulator parameter for the helical magnetic field with the amplitude $B_{u}$, period $\lambda_{u}$, and undulator frequency $\Omega_{u}=2 \pi c \beta_{\|} / \lambda_{u}, \Omega_{c}=e B_{0} / m c \gamma$ is the cyclotron frequency, and $\Delta=1-\Omega_{c} / \Omega_{u}$ is the

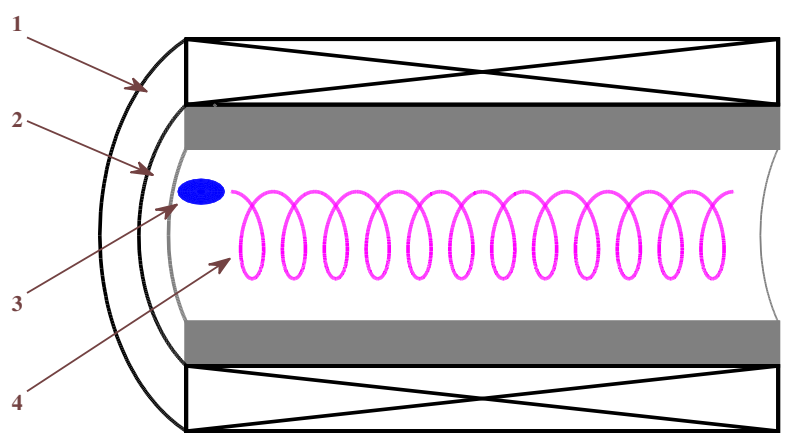

FIG. 1. Scheme of the radiation section: 1-magnetic system creating a transverse helical undulator and strong uniform longitudinal fields; 2 -oversized metal circular waveguide; 3 - electron bunch; 4-helical stationary electron trajectory.

relative mismatch of the resonance between free cyclotron and forced undulator particle oscillations. Correspondingly, the nonisochronity for longitudinal particle oscillations is described (within the ultrarelativistic approach) by the derivative

$$
\frac{\partial \beta_{\|}}{\partial \gamma}=\frac{1}{\gamma^{3}}\left(1+\frac{K^{2}}{\Delta^{3}}\right)
$$

The NMI for the longitudinal particle motion develops if this value is negative. This is realized when the two following conditions are fulfilled:

$$
\Delta<0, \quad|\Delta|^{3}<K^{2} .
$$

According to these conditions, the cyclotron frequency should be larger than the undulator frequency, $\Omega_{c}>\Omega_{u}$, but not too close to it (over-resonance regime).

The development of the NMI in an undulator has been already demonstrated in the one-dimensional approximation [9]. A significant increase in the radiation power was revealed in such a system. In the present work, excitation of terahertz radiation by short electron bunches is studied for an essentially more realistic model that takes into account finite transverse sizes of all elements of the radiation source: electron bunches, a helical undulator with uniform guiding magnetic field, and a circular operating waveguide (Fig. 1). The simulations were carried out using the original three-dimensional space-frequency code WB3D [20].

\section{METHOD OF SIMULATIONS AND RESULTS}

A detailed description of the self-consistent, spacefrequency approach for simulations of a pulsed relativistic electron beam propagation through a magnetostatic structure and its radiation emission is given in Ref. [20]. In the approach, the rf field excited in an operating waveguide is considered in the positive-frequency Fourier domain, which is followed by expansion of monochromatic Fourier field components in terms of transverse eigenmodes of a 
waveguide. The interaction between the field and "macroparticles" representing the electron beam is found from a self-consistent system of equations describing both the excitation and evolution of radiation along the interaction region, as well as motion of the particles.

The field affecting the particles and defining motion of the bunch in terms of the force equations includes both high-frequency and space-charge terms. A precise description of the space-charge field term which includes mutual electromagnetic interactions between relativistic charges moving arbitrarily in a waveguide appears to be a hard problem to solve. We use the "free-space" Lorenztransformed Coulomb field and neglect the effect of the waveguide walls on the space-charge field affecting the particles moving close to the waveguide axis [6,25]. The equations are integrated in the space-frequency domain along the beam line axis $z$, when each simulation particle $j$ arrives to a point $z$ at its own time $t_{j}(z)$. To obtain simultaneous positions of all the particles at the same moment $t_{i}(z)$ when a chosen particle $i$ arrives to a point $z$ (in order to calculate a rest-frame space-charge Coulomb field affecting the particle $i$ at this moment), a linear interpolation with constant velocities is used for all particles $j \neq i$ from their own times $t_{j}(z)$ to the moment $t_{i}(z)$. One can note that the field received in this procedure does not depend on the particles' accelerations; therefore, the resulted space-charge field obtained after Lorentz transformation back to the lab frame, seems to be equivalent to a folding of the quasi-Coulomb part of LiénardWiechert potential.

The approach was realized in the numerical code WB3D [20] and is used in the present work for analysis of $\mathrm{THz}$ coherent spontaneous undulator radiation produced in a metal waveguide by short and very dense electron bunches moving in a combined undulator field. A random Gauss distribution of 1,000 macroparticles is used in the simulations to introduce drive electron bunches. Parameters of the bunches (Table I) are close to the expected parameters

TABLE I. Basic parameters of the simulated THz source.

\begin{tabular}{ll}
\hline \hline Accelerator & \\
Type & Photoinjector rf-linac \\
Electron kinetic energy, $E_{k}$ & $6 \mathrm{MeV}(\gamma \approx 13)$ \\
Initial bunch duration, $T_{b}(\mathrm{rms})$ & $0.3 \mathrm{ps}\left(l_{b} \approx 0.1 \mathrm{~mm}\right)$ \\
Transverse bunch radius, $R_{b}(\mathrm{rms})$ & $0.5 \mathrm{~mm}$ \\
Waveguide & \\
Type & Circular cylindrical, metal \\
Radius & $5 \mathrm{~mm}$ \\
Dominant modes & $T E_{11}, T E_{12}, T M_{11}, T M_{12}$ \\
Helical undulator & \\
Period, $\lambda_{u}$ & $25 \mathrm{~mm}$ \\
Field amplitude, $B_{u}$ & $2 \mathrm{kG}$ \\
Undulator constant, $K_{u}$ & 0.47 \\
Uniform guiding field, $B_{0}$ & $75 \mathrm{kG}$ \\
\hline \hline
\end{tabular}

of the Israeli $\mathrm{THz}$ source (ITS) developed at Ariel University on the basis of a hybrid laser-driven photoinjector $[18,19]$.

In Ref. [9], an injection of a preformed 3-D bunch into adiabatically increasing undulator and constant guiding fields in free space has been studied. The simulations have confirmed that several undulator periods are sufficient to provide the injection without significant degradation in the quality of the electron bunch if the difference between the cyclotron and undulator frequencies is great enough, $|\Delta| \gtrsim 0.5$. In the present work, for the sake of simplification we consider the situation in which the bunch enters the combined magnetic fields so its center directly moves along a stationary helical trajectory. The methods of formation and injection of dense bunches will be the subject for detailed future work.

The NMI instability allows for a long-living existence of very dense and short electron bunches, which enables an intensive and efficient radiation emission. Though a planar undulator also can be used for realization of the NMI regime, it can lead to transverse drift and expansion of the bunch [14]. Because of it, unlike the configuration of the first stage of the experiments planned at the ITS [19], we consider the source with electron bunches moving in a helical undulator and radiating in a circular cylindrical waveguide. In order to maximally increase the radiation frequency and simplify a long distance bunch transport the operating waveguide with a large (oversized) cross section was chosen (Table I). In addition to the configuration replacements mentioned above, short electron bunches with charges of up to $5 \mathrm{nC}$ (much higher than it was supposed in the first-stage ITS design) are considered in the simulations.

When the guiding magnetic field is zero, the normalized transverse electron velocity is $0.47 \gamma_{0}^{-1}$ and a particle radiates in the chosen oversized waveguide at a frequency of about $3 \mathrm{THz}$. At the "positive" direction of the guiding magnetic field $\left(\Omega_{c}>0\right)$, the transverse velocity is significantly larger: for example, $\beta_{\perp}=1.2 \gamma_{0}^{-1}$ at a large over-resonance value $B_{u}=75 \mathrm{kG}$. This means that the longitudinal particle velocity and the Doppler-frequency shift in such a regime are essentially smaller in comparison with those at a zero guiding field. Correspondingly, the radiation frequency is reduced to $f_{s} \approx 1.5 \mathrm{THz}$. Very short electron bunches with the initial time duration of $T_{b}=0.3 \mathrm{ps}$, which is approximately equal to half of the radiation period, are considered. It is found that such bunches can coherently radiate in a long undulator due to the NMI.

At a relatively low or moderate charge, $q_{b}=0.5-1.5 \mathrm{nC}$, the characteristic duration of an initially short electron bunch is conserved or even turns to be a bit shorter during the bunch motion in the undulator thanks to the NMI [Figs. 2(a)-2(c)]. The destructive space-charge force grows with the charge, breaking the balance between 


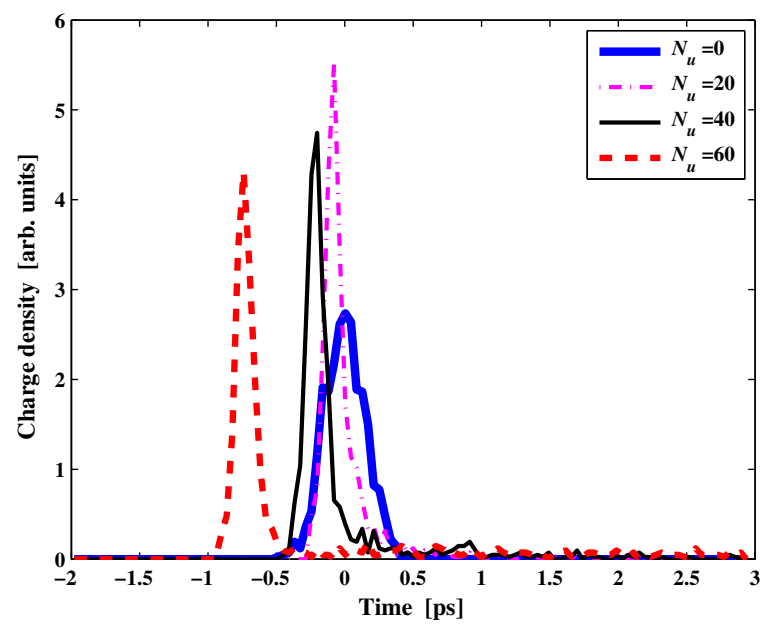

(a)

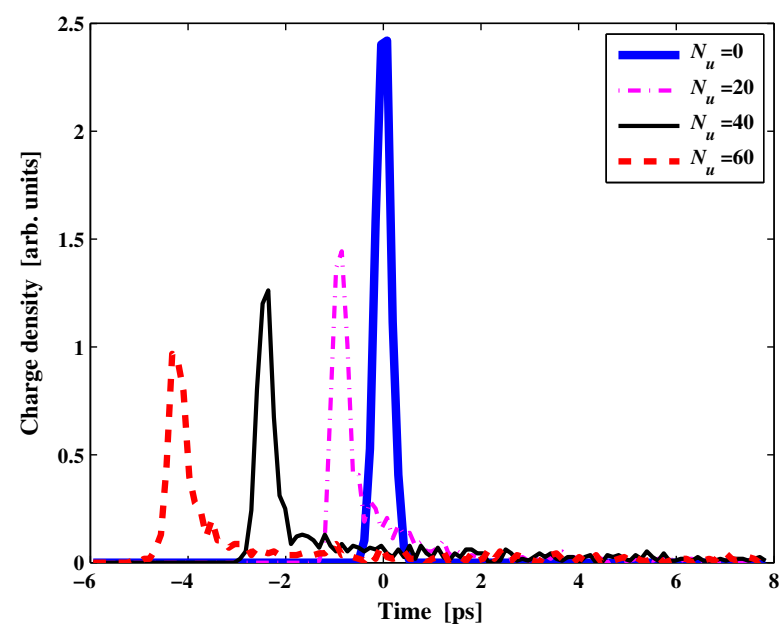

(c)

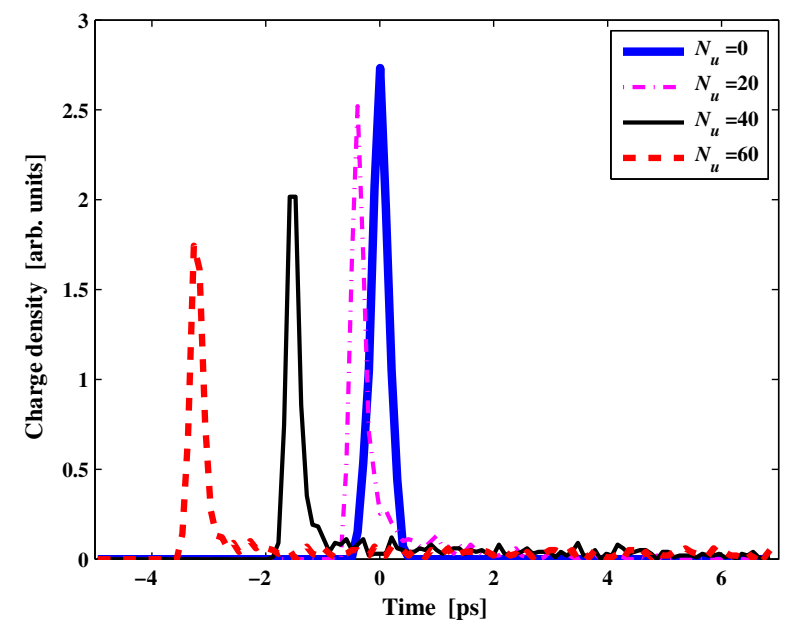

(b)

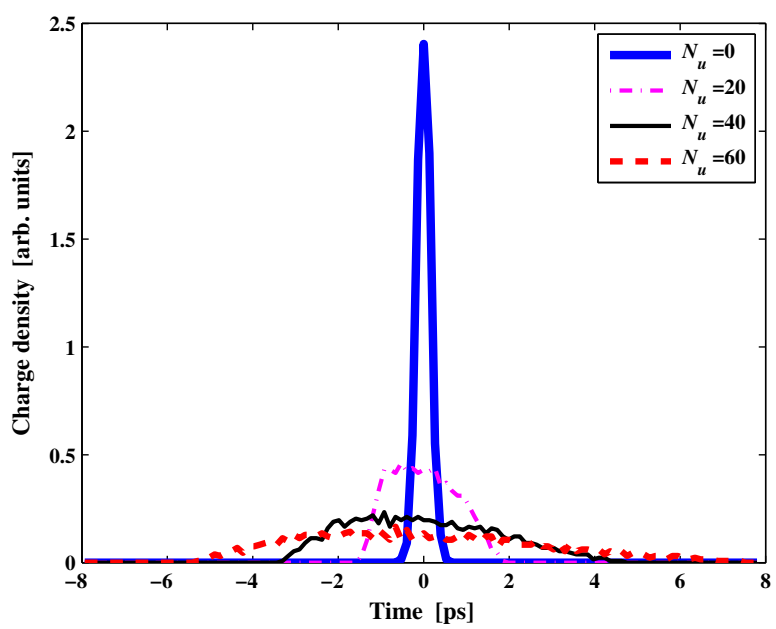

(d)

FIG. 2. Bunch density at the undulator entrance and after each 50 periods along the beam line: charges $0.5 \mathrm{nC}$ (a), $1.5 \mathrm{nC}$ (b), and $2.5 \mathrm{nC}$ (c) for the positive guiding magnetic field, and $0.5 \mathrm{nC}$ for the "negative" field (d). The time is given in the pictures relative to the "center of mass" of the bunch; presence of a bunch tail shifts the dominant peak of the bunch distribution out of the center.

the repulsive and attractive forces and reducing the NMI for charges higher than $2.5 \mathrm{nC}$. The main peak of the charge density is reduced and turns to be wider along the beam trajectory, while a considerable part of the charges leaves the central peak and forms a widely extended "tail" after it. In the case of an oppositely directed (negative) focusing magnetic field, the NMI is not developed at any charge, the bunch quickly expands, its duration grows monotonically [Fig. 2(d)] and the radiation is negligibly weak.

The mentioned features of the NMI in a undulator can be very clearly demonstrated on the example of a bunch consisting of several macroparticles (Fig. 3).

Compactness of the electron bunch can be also characterized along with the density by the so-called bunching factor $\left\langle\exp \left[i \omega_{s} t_{i}(z)\right]\right\rangle$, which characterizes bunch capability for the coherent radiation at the definite frequency $\omega_{s}$, where $t_{i}(z)$ is the motion time for the $i$ th particle to arrive at the position $z$, and the brackets stand for the statistical averaging over all the macroparticles presenting a bunch. With a relatively small or moderate charges $q_{b}<1.5 \mathrm{nC}$, the central peak of the bunch charge distribution remains short along tens of the undulator periods. A high bunching factor of more than $30 \%$ which is obtained for these charges even after 60 undulator periods (Fig. 4), manifests high coherence of the bunch radiation (coherent spontaneous emission). A strong space-charge repulsion leads to fast spreading of bunches with high charges $\left(q_{b}>2.5 \mathrm{nC}\right)$ and widens the central peak of their distribution. The bunching factor turns to be rather small (less than 10\%) in this situation like that for noncoherent spontaneous emission. The presence of a long tail of the bunch formed with low-energy electrons (Fig. 2) explains the evolution of bunch density and reducing the bunching factor (Fig. 4). These results are qualitatively consistent with the results of the general particle tracer (GPT) code simulations for a simpler model found in [9]. 


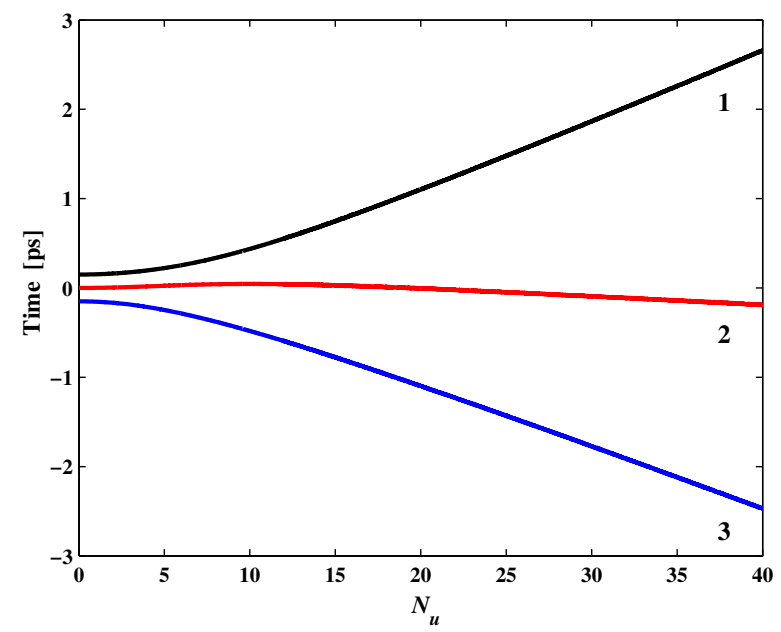

(a)

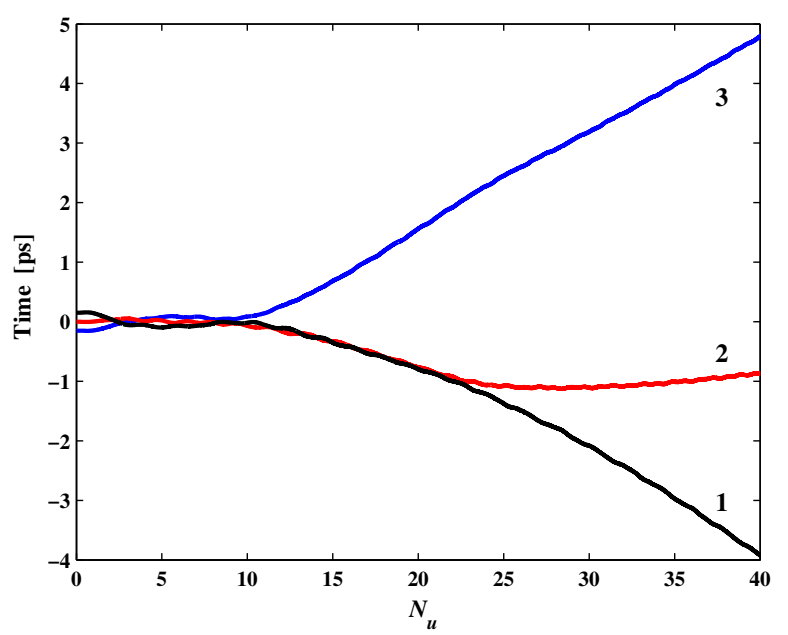

(b)

FIG. 3. NMI for three macrocharges of $0.5 \mathrm{nC}$ each, moving in the undulator. Fast spreading with the negative guiding field (a) and close motion of two particles presenting a compact "cluster" while the third particle moves away of them presenting a spreading "tail" in a strong positive guiding field (b).

The radius of the stationary helical trajectory $\rho=$ $K c /\left[\gamma_{0}\left(\Omega_{c}-\Omega_{u}\right)\right]=0.37 \mathrm{~mm}$ is much smaller than the $5 \mathrm{~mm}$ waveguide radius. Therefore, the electrons rotate very close to the waveguide axis, which gives rise to effective excitation of $T E_{11}, T E_{12}, T M_{11}$, and $T M_{12}$ waveguide modes only. Contributions of these modes are found to be of the same order of magnitude and overlap each other in frequency [Figs. 5(a)-5(c)]. With the undulator length, the spectrum peaks of the modes turn to be narrower, and the frequency overlap reduces. The amplitudes of other waveguide modes are negligibly small.

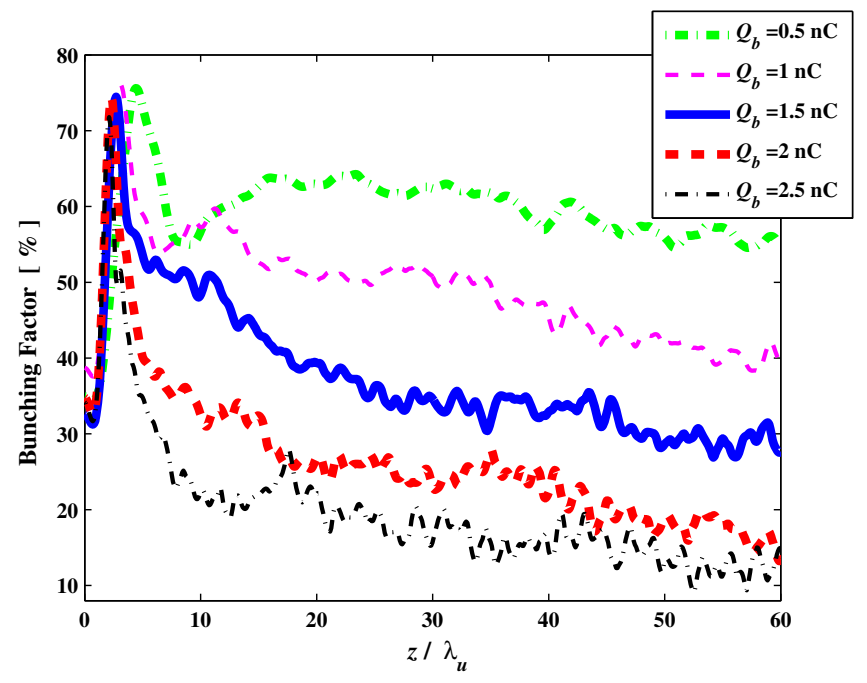

FIG. 4. Bunching factor at the central radiation frequency of $1.5 \mathrm{THz}$ as a function of number of undulator periods for various bunch charges.
The energy flux of spontaneous radiation is proportional to the bunch charge. To compare the radiation spectra emitted by bunches with different charges, the "normalized" spectral density $\left(1 / q_{b}\right) \mathrm{d} W / \mathrm{d} f$ of the $T E_{12}$ waveguide mode is also shown in Fig. 5(d).

The energy flux of the radiation $W(z)$ through a cross section $z$ is accumulated during the bunch propagation along the undulator [Fig. 6(a)]. The rate of accumulation can be characterized by the average radiation power $W(z) / \tau_{s p}$ through the cross section $z$, where $\tau_{s p}=$ $z /\left(c \beta_{\|}\right)-z / v_{g r}$ is the so-called slippage time and $v_{g r}$ is the group velocity of the radiation [Fig. 6(b)]. For low charges $q_{b} \leq 1 \mathrm{nC}$, the energy flux grows approximately linearly along the beam trajectory and the radiation power remains nearly constant. For high charges $q_{b}>2.5 \mathrm{nC}$, the energy flux seems to accumulate most of its energy at the very first undulator periods followed by a small growth at the rest of the bunch trajectory. The energy flux emitted by a lower charge can even overcome that of a higher charge [compare the results for 2.5 and $3.5 \mathrm{nC}$ in Fig. 6(a)]. Because of fast flux saturation at large charges, a very high radiation power is obtained just after a very short undulator: it reaches more than $1 \mathrm{GW}$ for $q_{b}=5 \mathrm{nC}$ after three undulator periods [Fig. 6(b)]. This high power corresponds to the radiation energy of $2.5 \mathrm{~mJ}$ in comparison with the initial bunch energy of $q_{b} E_{k}=30 \mathrm{~mJ}$, where $E_{k}=6 \mathrm{MeV}$ is the electron kinetic energy.

Due to the NMI, radiation emission grows remarkably at the beam charges less than $3.5 \mathrm{nC}$. Moreover, the simulations demonstrate an approximately constant energy flux being larger than $2 \mathrm{~mJ}$ for the charges in the interval $1.5-3.0 \mathrm{nC}$. The energy extraction efficiency is evaluated as the ratio of the radiation flux and the initial bunch energy $q_{b} E_{k}$ peaks at charges $1.0-1.5 \mathrm{nC}$, when more than $20 \%$ of 


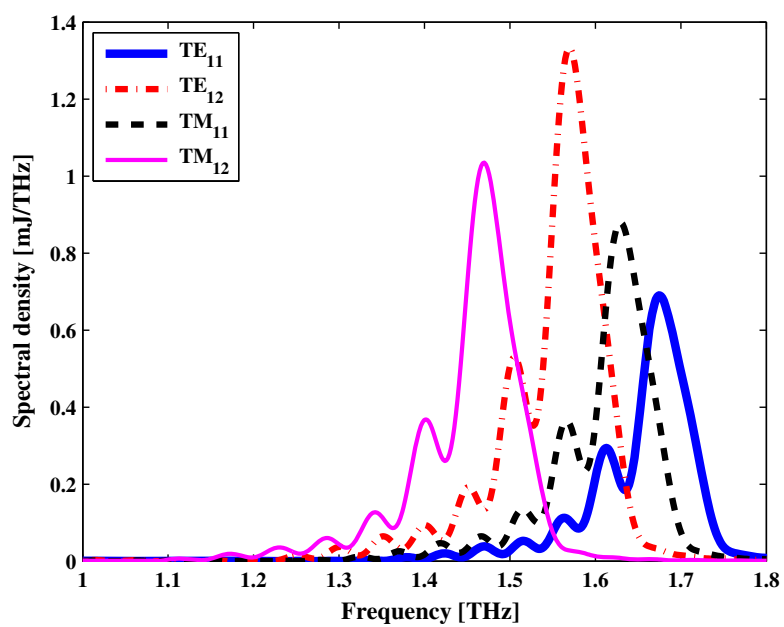

(a)

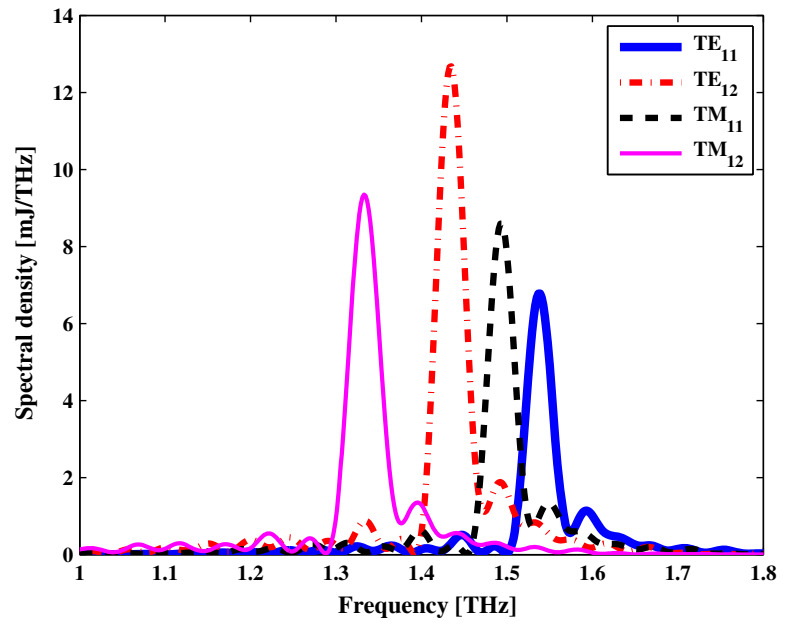

(c)

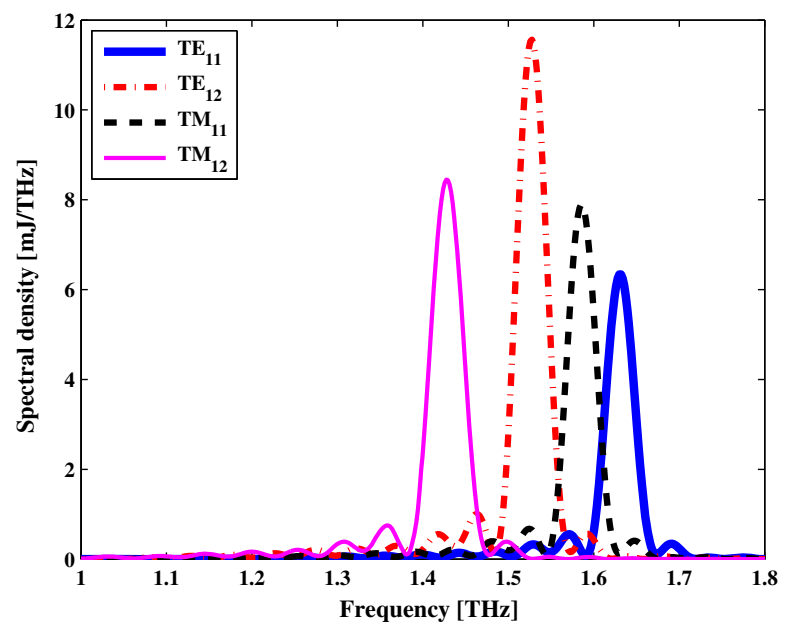

(b)

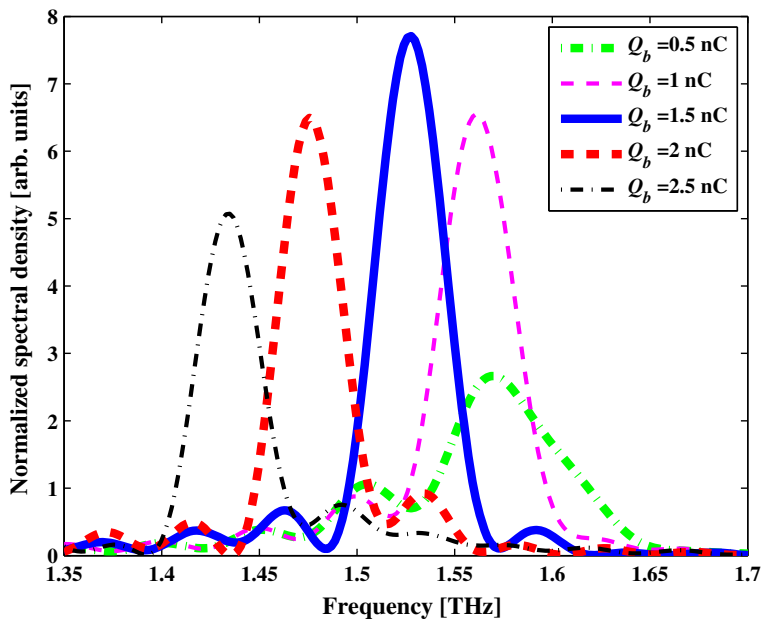

(d)

FIG. 5. Spectral radiation density $\mathrm{d} W / \mathrm{d} f$ for charges $0.5 \mathrm{nC}(\mathrm{a}), 1.5 \mathrm{nC}$ (b), and $2.5 \mathrm{nC}$ (c) obtained after 40 undulator periods. Normalized spectral density $\left(1 / q_{b}\right) \mathrm{d} W / \mathrm{d} f$ of the $T E_{12}$ waveguide mode (d) is given for comparison.

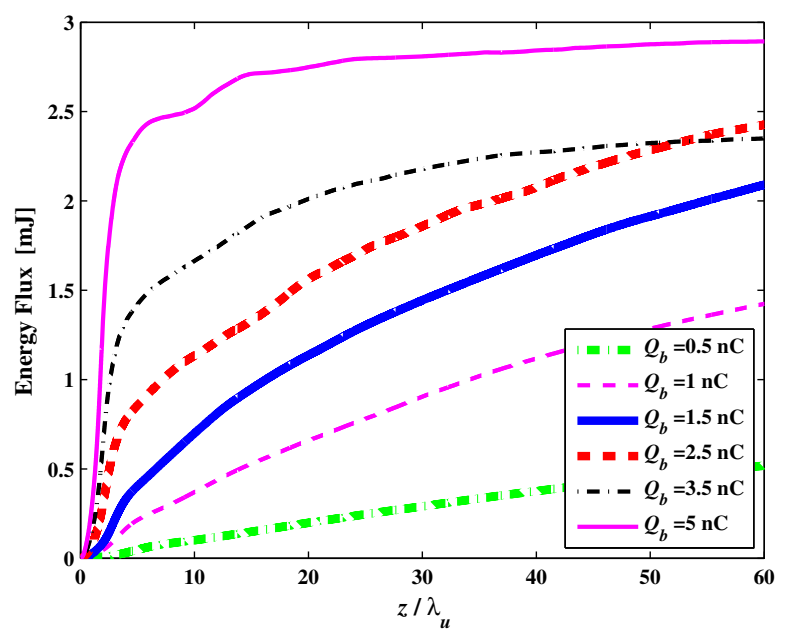

(a)

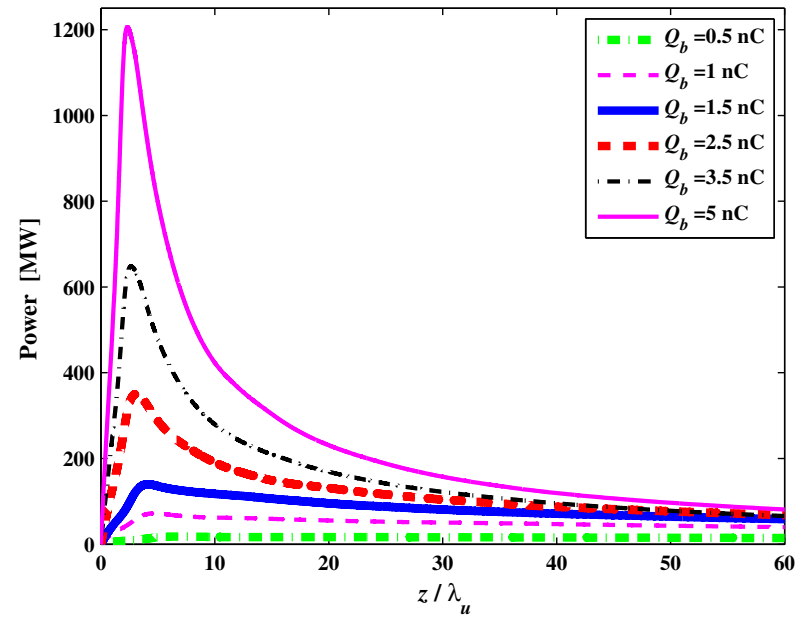

(b)

FIG. 6. Energy flux (a) and average power (b) along the beam line. 


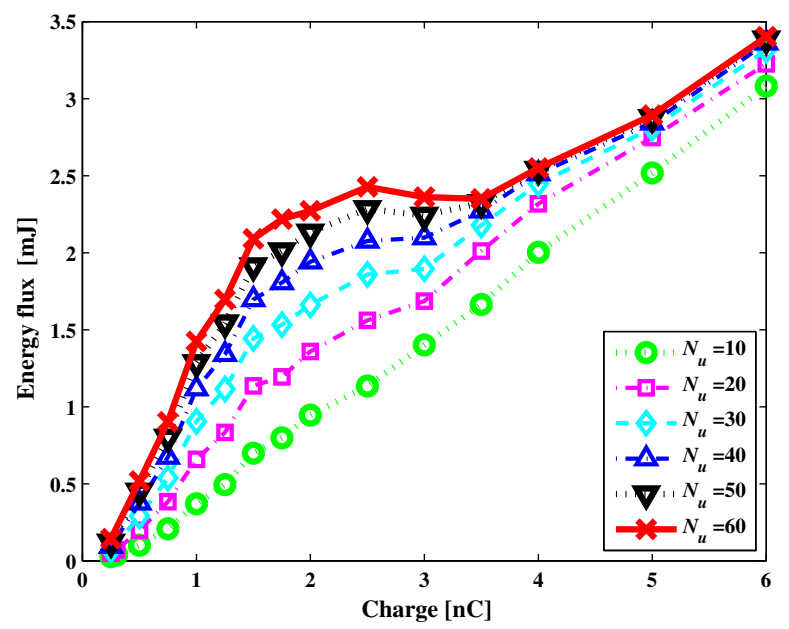

(a)

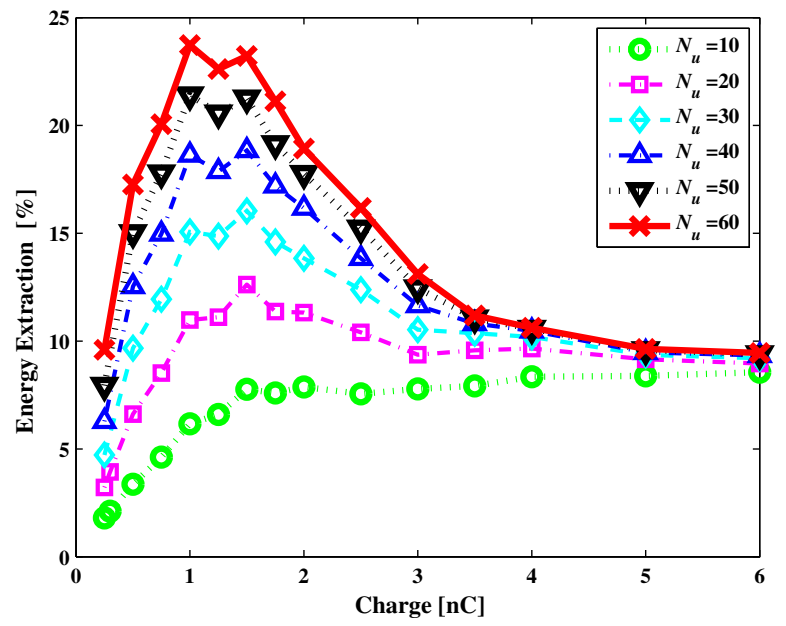

(b)

FIG. 7. Charge dependence of radiation energy flux (a) and energy efficiency (b).

the initial beam energy is converted into radiation (Fig. 7). At higher charges, the balance between the attraction caused by the nonisochronity and the direct Coulomb repulsion is gradually broken.

It should be noticed that the radiation efficiency can be found to be a somewhat lower than above the calculated value due to the influence of charges induced by the bunch in the waveguide metal walls and energy spread of the particles.

Because of the negative derivative $\frac{\partial \beta_{\|}}{\partial \gamma}$, the loss of the bunch energy in the radiation process leads to an increase in the longitudinal velocity and frequency of radiation during the pulse at low charges (Fig. 8). The corresponding frequency shift is as large as $70-140 \mathrm{GHz}$ at $q_{b}=0.5-1.0 \mathrm{nC}$. Presumably, this dependence can be used for some pump-probe methods. At higher charges, the radiation frequency is not changed significantly and then its increase is replaced with a decrease.

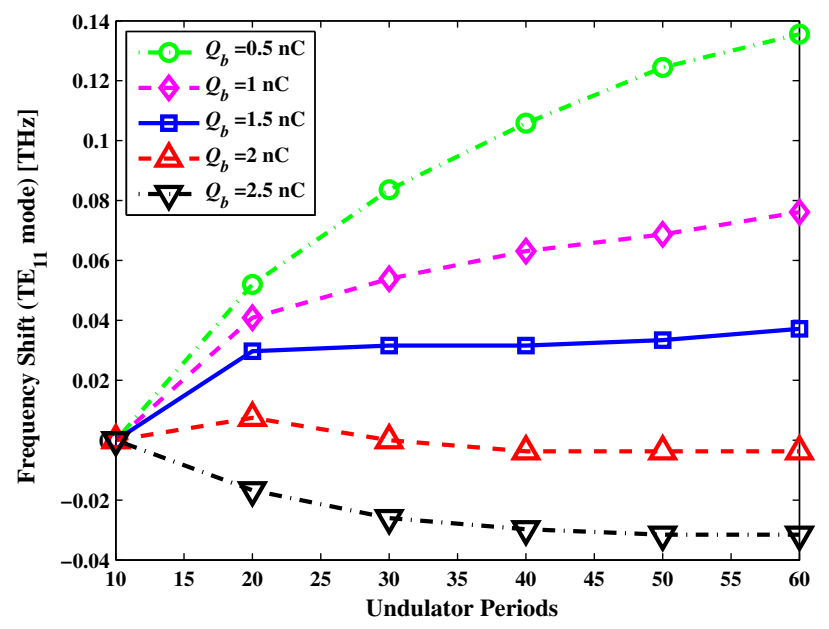

FIG. 8. Frequency shift of the radiation during the motion of the bunch.

\section{CONCLUSION}

The results of the simulations present a promising possibility for significant enhancement of extraction of coherent spontaneous undulator radiation from short and very dense electron bunches thanks to the NMI stabilization of their longitudinal sizes. Though the necessity of the very strong magnetic field complicates the design of a source essentially, it simultaneously ensures the transverse bunch confinement and makes almost trivial creation of the undulator field by means of redistribution of the guiding field by a helical iron insert [9]. Production of subpicosecond long, high charge density bunches for utilization in the radiation source discussed is a challenging task for accelerator physics. Our simulations demonstrate, however, that the effect of NMI is present even if longer bunches are used, although if resulting in some reduction of the system efficiency. Thus, the studied method opens up the way for realization of a relatively compact and very efficient source of narrowband terahertz pulses with milli-Joule energy and picosecond duration. The radiation frequency can be tuned in a wideband and is shifted remarkably during the pulse. The latter opportunity allows also realization of prospective pump-probe experiments by using terahertz and $\mathrm{x}$-ray pulses from the initial relatively low energy and final high-energy parts of accelerating electron bunches in large installations.

It seems that possibilities of creating very short electron bunches in laser-driven photoinjectors and then stabilizing its longitudinal size in undulators with an over-resonance guiding field may provide some additional success to the original ideas by Ginzburg and Motz [1,2].

\section{ACKNOWLEDGMENTS}

Two of the authors (V. L. B. and A. V.S.) are grateful for a partial support to the Russian Foundation for Basic Research, Project No. 16-02-00794 A. 
[1] V. L. Ginzburg, On radiation of micro-radio-waves and their absorption in air, Izv. Akad. Nauk SSSR, Ser. Fiz. II 165, 132 (1947).

[2] H. Motz, Applications of the radiation from fast electron beams, J. Appl. Phys. 22, 527 (1951).

[3] A. Doria, R. Bartolini, J. Feinstein, G. P. Gallerano, and R. H. Pantell, Coherent emission and gain from a bunched electron beam, IEEE J. Quantum Electron. 29, 1428 (1993).

[4] A. Gover, F. V. Hartemann, G. P. LeSage, N. C. Luhmann, R. S. Zhang, and C. Pellegrini, Time and Frequency Domain Analysis of Superradiant Coherent Synchrotron Radiation in a Waveguide Free-Electron Laser, Phys. Rev. Lett. 72, 1192 (1994).

[5] Yu. Lurie and Y. Pinhasi, Enhanced superradiance from energy-modulated short electron bunch free-electron lasers, Phys. Rev. ST Accel. Beams 10, 080703 (2007).

[6] Yu. Lurie, A. Friedman, and Y. Pinhasi, Single pass, THz spectral range free-electron laser driven by a photocathode hybrid rf linear accelerator, Phys. Rev. ST Accel. Beams 18, 070701 (2015).

[7] A. Gover, E. Dyunin, Y. Lurie, Y. Pinhasi, and M. V. Krongauz, Superradiant and stimulated-superradiant emission in prebunched electron-beam radiators. II: Radiation enhancement schemes, Phys. Rev. ST Accel. Beams 8, 030702 (2005).

[8] P. Musumeci, R. K. Li, and A. Marinelli, Nonlinear Longitudinal Space Charge Oscillations in Relativistic Electron Beams, Phys. Rev. Lett. 106, 184801 (2011).

[9] N. Balal, I. V. Bandurkin, V. L. Bratman, E. Magory, and A. V. Savilov, Negative-mass mitigation of Coulomb repulsion for terahertz undulator radiation of electron bunches, Appl. Phys. Lett. 107, 163505 (2015).

[10] H. P. Freund and P. Sprangle, Unstable electrostatic beam modes in free-electron-laser systems, Phys. Rev. A 28, 1835 (1983).

[11] A. K. Ganguly and H. P. Freund, Nonlinear analysis of free-electron-laser amplifiers in three dimensions, Phys. Rev. A 32, 2275 (1985).

[12] A. K. Ganguly and H. P. Freund, Three-dimensional simulation of the Raman free-electron laser, Phys. Fluids 31, 387 (1988).

[13] N. S. Ginzburg and N. Yu. Peskov, Non-linear theory of relativistic ubitrons with electron beams formed in an adiabatically increasing undulator field and a uniform longitudinal magnetic field, Tech. Phys. 58, 859 (1988).

[14] H. P. Freund and T. M. Antonsen, Principles of FreeElectron Lasers (Chapman and Hall, London, 1996).

[15] N. S. Ginzburg and N. Yu. Peskov, Nonlinear theory of a free electron laser with a helical wiggler and an axial guide magnetic field, Phys. Rev. ST Accel. Beams 16, 090701 (2013).

[16] I. V. Bandurkin, S. V. Kuzikov, and A. V. Savilov, Cyclotron-undulator cooling of a free-electron-laser beam, Appl. Phys. Lett. 105, 073503 (2014).

[17] I. V. Bandurkin, I. V. Osharin, and A. V. Savilov, Cyclotron radiation cooling of a short electron bunch kicked in an undulator with guiding magnetic field, Phys. Rev. ST Accel. Beams 18, 110702 (2015).

[18] E. Dyunin, Yu. Lurie, Y. Pinhasi, A. Gover, and H. Marks, A new THz FEL development project, IEEE 25th convention of electrical and electronics engineers in Israel, 2008, Eilat, Israel, p. 825.

[19] A. Friedman, N. Balal, E. Dyunin, Yu. Lurie, E. Magori, V. L. Bratman, J. Rosenzweig, H. Lay To, and A. Gover, Configuration and status of the Israeli $\mathrm{THz}$ free electron laser, in Proceedings of the 36th International Free Electron Laser Conference, Basel, Switzerland, 2014, p. 553.

[20] Y. Pinhasi, Yu. Lurie, and A. Yahalom, Space-frequency model of ultra-wide-band interactions in free-electron lasers, Phys. Rev. E 71 (2005), 036503.

[21] C. Nielsen and A. Sessler, Longitudinal space charge effects in particle accelerators, Rev. Sci. Instrum. 30, 80 (1959).

[22] A. A. Kolomensky and A. N. Lebedev, Stability of a charged beam in storage systems, At. Energ. 7, 549 (1959).

[23] V. L. Bratman, Instability of orbital motion in a layer of electrons rotating in a uniform magnetic field, Tech. Phys. 46, 2030 (1976).

[24] V. L. Bratman and A. V. Savilov, "Phase mixing" of bunches and decrease of negative-mass instability increments in cyclotron resonance masers, Phys. Plasmas 2, 557 (1995).

[25] Yu. Lurie and Y. Pinhasi, Modal description of longitudinal space-charge fields in pulse-driven free-electron devices, Phys. Rev. ST Accel. Beams 13, 050701 (2010). 